专家推介: 本文通过基于电子学取数的测试系统, 在地磁场环境对两种不同聚焦级结构的 MCP-PMT 进行了性 能测试，包括单光电子谱、后脉冲、渡越时间涨落、信号上升下降时间、收集效率等。另外并通过改变光电倍 增管与地磁场的相对位置，测得不同角度下 MCP-PMT 的性能，定性分析了地磁场对 MCP-PMT 的性能影响。

\title{
地磁场下微通道板型光电倍增管性能研究
}

\author{
苏德坦 ${ }^{2}$, 叶 皓 ${ }^{2}$, 孙建宁 ${ }^{1,2}$, 司曙光 ${ }^{2}$, 黄国瑞 ${ }^{2}$, 曹宜起 ${ }^{2}$, \\ 钱 森 ${ }^{3}$, 李 珅 $^{2}$, 王兴超 ${ }^{2}$, 任 玲 ${ }^{2}$, 马丽双 ${ }^{4}$
}

（1．微光夜视技术重点实验室，陕西 西安 710065；2．北方夜视技术股份有限公司，江苏 南京 211106;

3. 中国科学院高能物理研究所, 北京 $100049 ; 4$. 核探测与核电子学国家重点实验室, 中国科学技术大学, 安徽 合肥 230026）)

\begin{abstract}
摘要: 通过基于电子学取数的测试系统，在地磁场环境对两种不同聚焦级结构的微通道板型光电倍增 管 (microchannel plate type photomultiplier tubes, MCP-PMT) 进行了各项性能测试, 例如单光电子谱、 后脉冲、渡越时间涨落、信号上升下降时间、收集效率等。并通过改变光电倍增管与地磁场的相对位 置, 测得不同角度下 MCP-PMT 的性能, 并且经对比屏蔽磁场条件下与地磁场环境下 MCP-PMT 的性 能, 定性分析了地磁场对 MCP-PMT 的性能影响。
\end{abstract}

关键词: 微通道板型光电倍增管; 地磁场; 聚焦级结构

中图分类号：O472 文献标识码：A 文章编号：1001-8891(2020)08-0742-05

\section{Performance of the Microchannel Plate Type Photomultiplier Tube Under a Geomagnetic Field}

\author{
SU Detan ${ }^{2}$, YE Hao ${ }^{2}$, SUN Jianning ${ }^{1,2}$, SI Shuguang ${ }^{2}$, HUANG Guorui ${ }^{2}$, \\ CAO Yiqi ${ }^{2}$, QIAN Sen ${ }^{3}$, LI Shen ${ }^{2}$, WANG Xingchao ${ }^{2}$, REN Ling ${ }^{2}$, MA Lishuang ${ }^{4}$ \\ (1. Science and Technology on Low-Light-Level Night Version Laboratory, Xi'an 710065, China; \\ 2. North Night Vision Technology CO. LTD, Nanjing 211106, China; \\ 3. Institute of High Energy Physics, Chinese Academy of Sciences, Beijing 100049, China;
}

4. State Key Laboratory of Particle Detection and Electronics, University of Science and Technology of China, Hefei 230026, China)

\begin{abstract}
Using the electronic data acquisition system, various performance tests of microchannel plate type photomultiplier tubes (MCP-PMT) at different focus levels were performed in the geomagnetic environment, such as single photoelectron spectrum and after pulse, transit time fluctuations, signal rise and fall time, collection efficiency and so on. By changing the relative position of the photomultiplier tube and the geomagnetic field, the performance of the MCP-PMT at different angles was measured, and the geomagnetic field was qualitatively analyzed by comparing the performance of MCP-PMT under the shielding magnetic field and geomagnetic field.
\end{abstract}

Key words: MCP-PMT, geomagnetic field, focusing structure

\section{0 引言}

1911 年, 奥地利物理学家 V. F. Hess 用 3 台静电 计测得 $5300 \mathrm{~m}$ 的高空上电离率增长到地面的 4 倍。 他认为该观察结果最好的解释是设想一种高穿透力
的射线从宇宙进入大气层。后人将这种射线命名为宇 宙线, V.F. Hess 据此获得 1936 年诺贝尔物理学奖。 虽然当前对宇宙线的起源尚无完备的理论，但人类已 意识到某些宇宙线的能量远超目前人工加速器所能 
达到的水平。为通过宇宙线解决当前物理学界遇到的 新问题, 我国发改委批准建立高海拔宇宙线观测站 ( Large High Air Altitude Shower Observatory, LHAASO），旨在探索高能宇宙线起源以及相关的宇 宙演化、高能天体演化和暗物质等前沿问题。 LHAASO 项目采用多种探测手段实现复合、精确的测 量, 大幅度提高灵敏度, 覆盖更宽广的能谱, 从而建 设第三代 $\gamma$ 天文探测器 ${ }^{[1]}$, 这就要求光电倍增管具有较 好的渡越时间离散值和低暗计数。针对 LHAASO 项 目对光电倍增管的特殊要求, 北方夜视技术股份有限 公司设计出一种扩张结构微通道板型光电倍增管。

LHAASO 项目中, 光电倍增管的核心指标是光电 阴极不同位置出射光电子的渡越时间涨落 (transit time spread, 以下简称 TTS), TTS 主要受光电倍增 管结构和分压器电路的影响。通过 CST 仿真软件计算 分析, 为了提高 TTS, 可以通过增加阴极和聚焦级之 间的电压差或者扩大聚焦级直径实现, 而阴极与聚焦 级之间的电压差不能过高, 因此只能通过扩大聚焦级 直径来解决。为此我们专门设计出一种高稳定性的自 动扩张型聚焦级, 在不改变过渡节口径的情况下大幅 增大了聚焦级直径。为优化信号上升时间和信号下降 时间, 并降低噪声和 TTS, 在原分压器的基础上优化 目前的分压比。

微通道板的每个孔径约为 $20 \mu \mathrm{m}$, 孔内壁使用电 阻和二次发射涂层处理, 当孔壁被二次电子轰击时, 每个微孔像单独的电子倍增器一样出现电子倍增现 象, 电子倍增的具体状态因入射电子的初始能量和入 射角度的不同而发生改变。当光电倍增管在磁场中工 作时, 由于同时存在电场和磁场, 所以电子轨迹同时 受到电场力及洛仑兹力的作用, 并出现相应变化 ${ }^{[2]}$ 。

传统的小尺寸微通道板型光电倍增管理论上可 以在高达 $2 \mathrm{~T}$ 的磁场强度下工作, 这是因为微通道板 有优秀的抗磁场干扰能力。而在大尺寸光电倍增管 中, 光电子沿较长的路径运动, 此时电子轨迹很容易 受到轻微的地磁场的影响, 从而导致收集效率发生变 化。变化的主要原因是光电子不能准确聚焦到第一 MCP 的位置, 且较小的 MCP 开口也使光电子更容易 受到地磁场的影响 ${ }^{[3]}$ 。

为研究光电倍增管在地磁场中的最佳相对摆放 位置, 本文对光电倍增管结构受地磁场影响和光电倍 增管安装位置受地磁场影响分别进行不同角度的研 究, 最终得到最佳相对摆放位置, 并通过扩张结构与 非扩张结构光电倍增管在地磁场下性能对比, 得到扩 张型聚焦级和新分压器对地磁场下工作性能的影响。

\section{1 测试环境及测试方法}

\section{1 测试环境}

\subsection{1 温度湿度及光量子数}

所有实验均在温度 $22^{\circ} \mathrm{C} \pm 3{ }^{\circ} \mathrm{C}$, 湿度小于等于 $60 \%$ 的条件下进行。暗室内部全部黑化, 关灯、关门 后暗室内照度小于 $10^{-12} \mathrm{~lx}$ （20 英寸光电倍增管置于 其中, 暗室计数率增量小于 $100 \mathrm{~Hz}$, 光子数小于 100 个 $/ \mathrm{m}^{2}$ )。使用 $405 \mathrm{~nm}$ LED (light emitting diode) 作 为光源, 光源散射成圆雉面并照射到光电倍增管阴极 面上, 测试过程中保证温湿度与光强基本恒定。

\subsection{2 屏蔽环境}

北方夜视技术股份有限公司建造了能同时屏蔽 高频电磁场和低频电磁场的屏蔽室。磁屏蔽室主体由 屏蔽层和主体骨架组成, 通过屏蔽焊接工艺, 形成一 个完整的密封多面体。屏蔽层采用 $6 \mathrm{~mm}$ 纯铁板（六 面一致），通过屏蔽焊接构成连续的四面体。对磁屏 蔽室内的磁场进行实地测量, 磁场强度集中分布 10 $\mathrm{mGs} \sim 20 \mathrm{mGs}$ 之间, 可见磁屏蔽室具有良好的地磁屏 蔽与电磁屏蔽效果。使用光纤为光电倍增管提供光 源, 并使用电子学取数系统测试光电倍增管相关性 能。

\subsection{3 地磁场环境}

本实验在东经 $118^{\circ} 46^{\prime}$ 、北纬 $31^{\circ} 54^{\prime}$ 附近进行, 在该地测量地磁场, 磁场强度大约为 $450 \mathrm{mGs}$ 。在地 磁场环境下, 需要重新搭建一套测试装置, 该设备 在搭建过程中, 已注意远离铁、镍等对磁场敏感的 金属材料。将待测光电倍增管放置在黑色长筒中, 该长筒顶端装有光纤固定装置。使用光纤为光电倍 增管提供光源, 并使用电子学取数系统测试光电倍 增管相关性能。

\section{2 测试方法}

\subsection{1 工作电压}

信号发生器输出两路同步脉冲信号, 信号频率 1

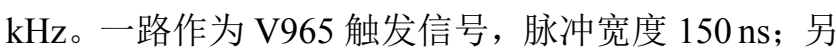
一路作为激光二极管 (以下简称 LD) 的电源, 脉冲 宽度 $10 \mathrm{~ns}$ 。调节 LD 脉冲驱动宽度, 使 LD 发光 10 次, 光电倍增管只探测到一次信号。将阳极输出信号 放大到 N979 放大器, 经过放大的信号输送到 V965 测试单光电子谱。使用双高斯函数分别拟合单光电子 谱的台阶峰和信号电荷峰, 台阶峰拟合区间为 $\mu-$ $1 \sigma \sim \mu+1.5 \sigma, \mu$ 为峰位值, $\sigma$ 为标准差; 信号电荷峰 拟合区间为 $\mu-1 \sigma \sim \mu+1 \sigma, \mu$ 为峰位值， $\sigma$ 为标准差。 增益由下式得到: 


$$
G=\frac{\left(X_{\text {sig }}-X_{\text {ped }}\right) \cdot q}{\mathrm{e}}
$$

式中: $X_{\mathrm{sig}}$ 为单光电子信号谱的拟合峰位; $X_{\mathrm{ped}}$ 为电子 学台阶的拟合峰位; V965 的 $q$ 值为 $25 \mathrm{fC}$; $\mathrm{e}$ 为单电 子电荷量。当增益达到 $1 \times 10^{7}$ 时, 程控高压电源显示 的电压值即为光电倍增管的工作电压。

\subsection{2 单光电子峰谷比}

单光单子峰谷比指在单光电子输入状态下, 脉冲 信号的峰值计数值与台阶峰和信号峰之间的谷位置 计数值的比值。使用高斯函数拟合测试工作电压时测 得的单光电子信号电荷谱, 取最低点及其前后各 2 点, 共 5 个点的均值, 每个点电荷量为 $25 \mathrm{fC}$ 。拟合区间 为 $\mu-1 \sigma \sim \mu+1 \sigma, \mu$ 为峰位值, $\sigma$ 为标准差。单光子 峰谷比 $P / V$ 由下式得到:

$$
P / V=N_{P} / N_{V}
$$

式中: $N_{\mathrm{P}}$ 为拟合函数的幅值; $N_{\mathrm{V}}$ 为谷位置的高度值。

\subsection{3 渡越时间涨落}

渡越时间指入射光入射到光阴极面起, 到输出脉 冲出现为止的时间。渡越时间涨落为所有单光子脉冲 渡越时间的起伏。信号发生器输出两路同步脉冲信 号, 信号频率 $1 \mathrm{kHz}$ 。一路作为 V1290A 触发信号, 脉冲宽度 $150 \mathrm{~ns}$; 另一路作为 $\mathrm{LD}$ 驱动电源, 脉冲宽 度 $10 \mathrm{~ns}$ 。计算光纤衍射角并调整光纤端头与光电倍增 管的距离, 使光纤输出的光覆盖光电倍增管阴极面的 直径为 $460 \mathrm{~nm}$ 。调节高压电源输出电压值, 使光电倍 增管增益为 $1 \times 10^{7}$ 。调节 LD 驱动脉冲幅度, 使 LD 发光十次, 光电倍增管只探测到一次光电信号。将阳 极输出信号输送到 N979 放大器, 经过放大的信号输 送到 V1290A 进行时间测试, 测试数据使用高斯函数 拟合, 下式即为 TTS 的计算公式:

$$
\mathrm{TTS}=\left[(2.35 \sigma)^{2}-T^{2}\right]^{1 / 2}
$$

式中: $\sigma$ 为拟合函数的标准差; $T$ 为测试系统的 TTS 值（ $T$ 使用 TTS 小于 $0.5 \mathrm{~ns}$ 的光电倍增管标定）。

\subsection{4 信号上升下降时间}

信号上升时间 $t_{\mathrm{u}}$ 为信号前沿从幅度值的 $10 \%$ 上升 到 $90 \%$ 的时间, 信号下降时间 $t_{\mathrm{d}}$ 为信号后沿从幅度值 的 $90 \%$ 下降到 10\%的时间。使用信号发生器输出两路 同步脉冲信号，信号频率 $1 \mathrm{kHz}$ 。一路作为示波器的 触发信号, 脉冲宽度 $150 \mathrm{ns;}$ 另一路作为 LD 驱动电 源, 脉冲宽度 $10 \mathrm{~ns}$ 。调节高压电源输出电压值, 使光 电倍增管增益为 $1 \times 10^{7}$, 调节 LD 驱动脉冲幅度, 使 $\mathrm{LD}$ 发光 10 次, 光电倍增管只探测到一次光电信号。 将阳极输出信号输送到示波器, 分别测试大量波形的
上升时间与下降时间, 并通过高斯函数拟合, 拟合函 数的中心值作为该光电倍增管的信号上升时间值与 下降时间值。

\section{2 测试结果与分析}

\section{1 光电倍增管安装位置不同带来的影响}

将光电倍增管的尾部沿中心轴指向光阴极为正 方向, 将该方向与地磁场场强的夹角作为光电倍增管 的相对方向。当光电倍增管安装位置不同, 即相对方 向不同时, 其内部电场场强相对于地磁场场强的位置 发生变化。光电子在光电倍增管内部受到的电场力及 洛仑兹力也会随之发生变化, 因此随光电倍增管安装 位置不同, 光电子的运动轨迹也会不同, 使光电倍增 管在不同位置表现出不同的性能 ${ }^{[4]}$ 。

扩张结构光电倍增管与非扩张结构光电倍增管 相比, 主要是聚焦级结构发生了改变, 以下实验在相 同安装位置下分别测试扩张结构型与非扩张结构型 光电倍增管在地磁场环境下性能的变化。

\section{2 地磁场下光电倍增管各性能变化}

\subsection{1 增益}

光电子被聚焦级有效收集后, 会在微通道板内倍 增, 经微通道板倍增放大的电流信号从阳极输出。我 们使用了屏蔽条件下增益为 $1 \times 10^{7}$ 的固定高压, 并将 安装位置倾斜角由 $0^{\circ}$ 转到 $330^{\circ}$ 。从图 1 中可以看出, 两种结构的光电倍增管在没有屏蔽时, 增益均表现出 一定的角度依赖性。非扩张结构与扩张结构相比, 增 益最大变化值均在 $\pm 10 \%$ 左右, 但相同旋转角度下, 大小变化并不相同, 聚焦级结构变化导致光电倍增管 中电场分布不同, 电子运行轨迹发生改变, 击打到通 道板上能量、速度方向均发生改变, 对增益在地磁场 下的性能表现有一定影响。

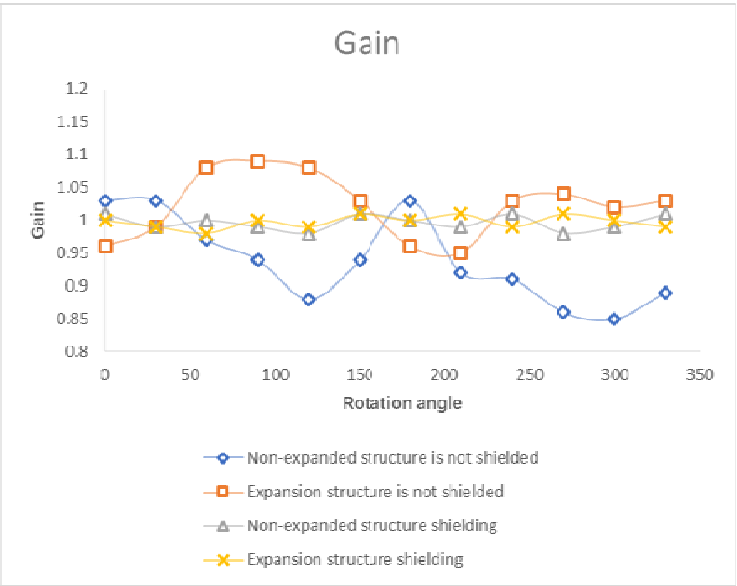

图 1 增益变化

Fig.1 Gain variation 


\subsection{2 峰谷比}

微通道板 ALD（atomic layer deposition）涂层的 二次发射产率与初级电子的能量和入射角度有关, 初 级电子能量过低或过高都会降低 ALD 涂层的二次发 射产率。从图 2 中可以看出, 光电子在倍增管内飞行 时运动轨迹受到磁场影响, 运行轨迹的集中性严重变 差, 导致峰谷比与屏蔽条件下相比显著降低。

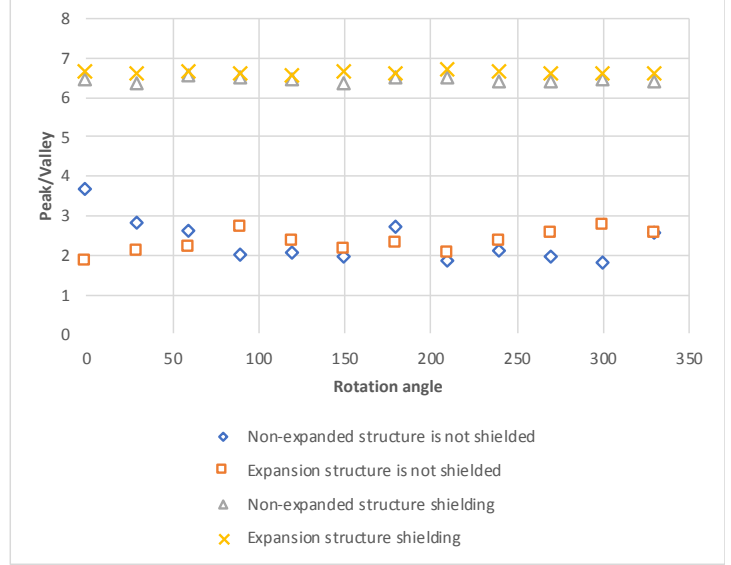

图 2 峰谷比变化

Fig.2 Peak-to-valley variation

\subsection{3 能量分辨率}

光电倍增管的能量分辨率 $\mathrm{ER}=\sigma / G_{\text {peak }}$, 其中 $\sigma$ 为 单光子峰高斯分布的半高宽, $G_{\text {peak }}$ 为单光子高斯分布 的中心道址, 光电倍增管的能量分辨率与入射粒子的 能量有关, 电磁场发生改变时, 电子运行轨迹不同, 受电场加速情况不同, 电子能量相应改变。图 3 中给 出扩张结构与非扩张结构光电倍增管在地磁场环境 下能量分辨率的变化。

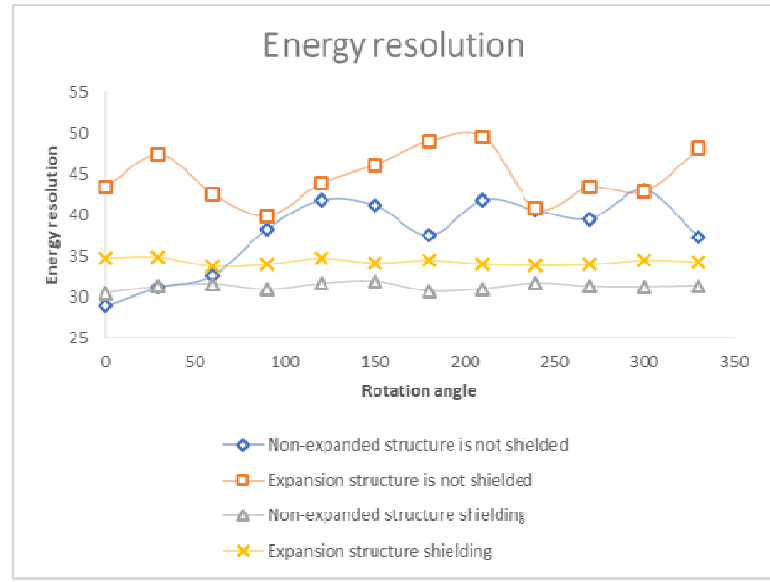

图 3 能量分辨率变化

Fig.3 Energy resolution variation

2.2.4 渡越时间涨落

光电子运动轨迹会直接影响光电子的飞行时间, 因此光电倍增管的渡越时间与光电子的运动轨迹密
切相关, 电子由于初始出射方向不同, 地磁场下电子 轨道会随出射方向不同而改变, 会对光电倍增管的 TTS 产生较大影响 ${ }^{[5]}$ 。从图 4 中可以看到, 扩张结构 对 TTS 有很明显的改善, 而在相同旋转角度下, 两种 结构的光电倍增管 TTS 变化表现相似, 在垂直于磁场 强度方向, TTS 所受影响较小。但与无屏蔽条件相比, 两种光电倍增管的 TTS 性能均发生明显恶化。

\subsection{5 信号上升时间}

与打拿极相比, 微通道板型大大减少了光电子在 倍增级的飞行时间。微通道板的厚度在 $0.5 \sim 1 \mathrm{~mm}$ 之 间, 光电子在很短的时间内就可以达到 $10^{7}$ 的量级, 因此地磁场对信号上升时间影响较小。从图 5 中可以 看出, 暴露在地磁场下的光电倍增管信号上升时间略 有升高，但幅度并不显著。

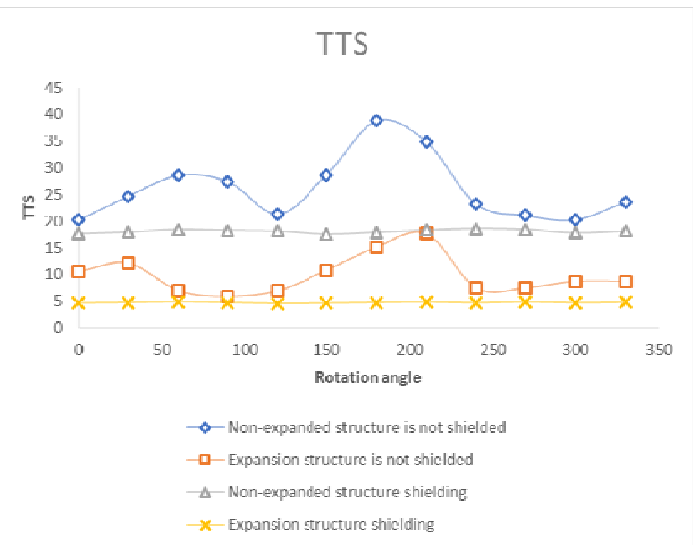

图 4 渡越时间变化

Fig.4 TTS variation

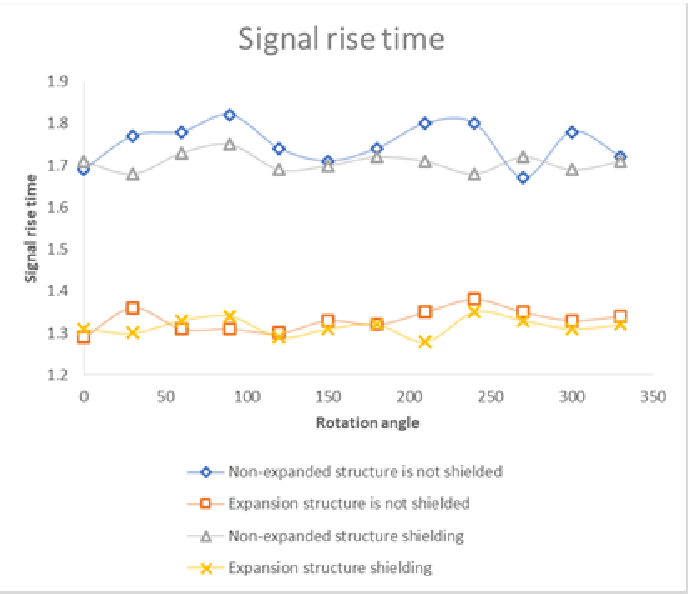

图 5 信号上升时间变化

Fig.5 Signal rise time variation

\subsection{6 信号下降时间}

从图 6 中可以看出，与信号上升时间相同，由于 光电子在倍增级的时间很小, 暴露在地磁场下的光电 倍增管信号下降时间略有升高，但幅度并不显著。 


\subsection{7 收集效率}

收集效率与电子飞行轨迹直接相关, 光电子从 光阴极飞出后, 在聚焦电场作用下, 向聚焦级运动, 被聚焦级有效收集的光电子才能被光电倍增管倍 增。由于地磁场发生改变, 能飞行到微通道板上的 电子数目会相应减少, 能被倍增的电子也随之减少。 从图 7 中可以看出, 收集效率表现出很强的角度依 赖性, 在极端角度下, 扩张结构型光电倍增管收集 效率衰减很大。

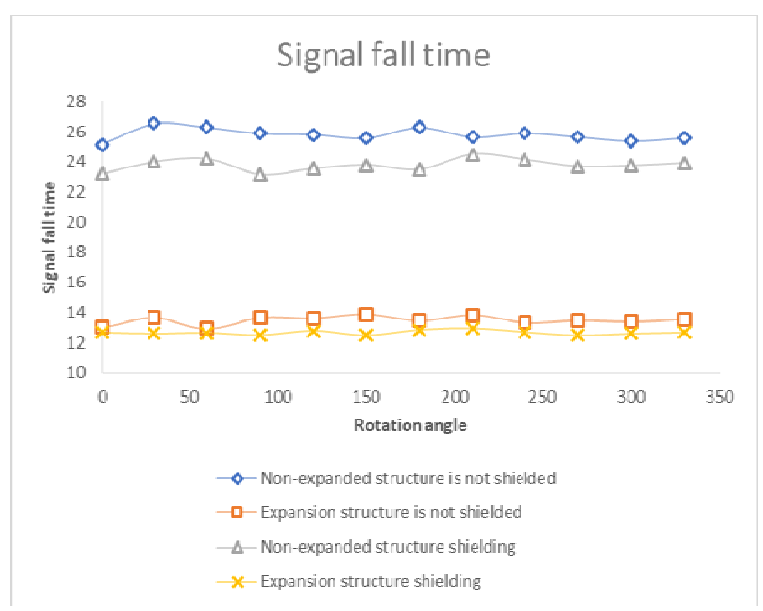

图 6 信号下降时间变化

Fig.6 Signal fall time variation

\section{参考文献:}

[1] ZONG Zizhao. Study of cosmic rays by Auger and LHAASO: R\&D and Data Analysis of Auger Prime and simulations for LHAASO[D]. Paris: Université Paris-Saclay, 2018.

[2] 王文文. 大面积光电倍增管地磁场性能影响和寿命老化研究[D]. 南 京: 南京大学, 2015.

WANG Wenwen. The Research on Geomagnetic Effect and Aging Performance of Large Area MCP-PMT[D]. Nanjing: Nanjing University, 2015 .

\section{3 结论}

本文主要讲述了两种不同结构的微通道板型光 电倍增管在地磁场下的性能表现, 并研究了多个性能 与摆放角度的关联性。由于体积较大, 地磁场对大尺 寸光电倍增管的性能影响不能忽略, 同时由于聚焦级 的结构不同, 不同类型的光电倍增管受地磁场的影响 也有差异。为保证光电倍增管在最佳状态下工作，应 建设磁屏蔽系统, 否则需要通过实验或理论计算找到 相对地磁场光电倍增管的最佳摆放位置。

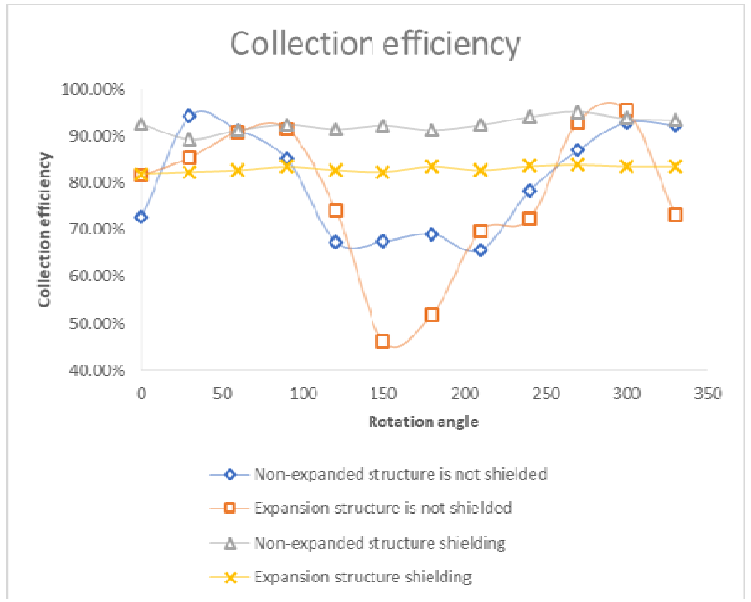

图 7 收集效率变化

Fig.7 Collection efficiency variation

[3] Rieke J, Belias A, Dzhygadlo R, et al. Resolution changes of MCP-PMTs in magnetic fields[J]. Journal of Instrumentation, 2016, 11(5): C05002.

[4] LIAO Donghao, LIU Hongbang, ZHOU Yixiong, et al. Study of TTS for a 20-inch dynode PMT[J]. Chinese Physics C, 2017, 41(7): 127-132.

[5] CHEN P, TIAN J, ZHAO T, et al. Design of the large area MCP-PMT[J]. Nuclear Instruments and Methods in Physics Research Section A Accelerators Spectrometers Detectors and Associated Equipment, 2018, 912: 163-166. 